\title{
Intégrer la littérature jeunesse en classe à l'aide de réseaux littéraires
}

\section{Using Literary Networks to Integrate Children's Literature into the Classroom}

\section{Cómo integrar la literatura juvenil en clase a través de las redes literarias}

\author{
Marie Dupin de Saint-André, Isabelle Montésinos-Gelet et Robert Bourdeau
}

Volume 61, numéro 1, janvier-mars 2015

URI : https://id.erudit.org/iderudit/1029001ar

DOI : https://doi.org/10.7202/1029001ar

Aller au sommaire du numéro

Éditeur(s)

Association pour l'avancement des sciences et des techniques de la documentation (ASTED)

ISSN

0315-2340 (imprimé)

2291-8949 (numérique)

Découvrir la revue

Citer cet article

Dupin de Saint-André, M., Montésinos-Gelet, I. \& Bourdeau, R. (2015). Intégrer la littérature jeunesse en classe à l'aide de réseaux littéraires. Documentation et bibliothèques, 61(1), 22-31. https://doi.org/10.7202/1029001ar
Résumé de l'article

Pour intégrer la littérature jeunesse en classe de manière à soutenir les compétences langagières et disciplinaires des élèves, le recours aux réseaux littéraires s'avère précieux. Dans cet article, nous explorons ce type de dispositif, qui repose sur la mise en lien d'oeuvres littéraires, en décrivant d'abord différents types de réseaux, en présentant ensuite la manière dont un enseignant planifie son année scolaire en organisant les tâches confiées aux élèves autour de réseaux variés et, enfin, en mettant en lumière divers obstacles à leur création ainsi que des pistes pour aider les enseignants à les surmonter.
Tous droits réservés (c) Association pour l'avancement des sciences et des techniques de la documentation (ASTED), 2015
Ce document est protégé par la loi sur le droit d'auteur. L'utilisation des services d'Érudit (y compris la reproduction) est assujettie à sa politique d'utilisation que vous pouvez consulter en ligne. 


\section{Intégrer la littérature jeunesse en classe à l'aide de réseaux littéraires}

\author{
MARIE DUPIN \\ DE SAINT-ANDRÉ \\ Coordonnatrice du Centre \\ de diffusion et de formation \\ en didactique du français \\ de l'Université de Montréal \\ marie.dupin.de.saint.andre \\ @umontreal.ca
}

\author{
ISABELLE \\ MONTÉSINOS-GELET \\ Professeure, Université \\ de Montréal \\ isabelle.montesinos.gelet \\ @umontreal.ca
}

\author{
ROBERT \\ BOURDEAU \\ Enseignant, \\ Commission scolaire \\ de la Vallée-des-Tisserands \\ bourdeaur@csvt.qc.ca
}

\section{RÉSUmÉ | ABSTRAct | RESUMEN}

Pour intégrer la littérature jeunesse en classe de manière à soutenir les compétences langagières et disciplinaires des élèves, le recours aux réseaux littéraires s'avère précieux. Dans cet article, nous explorons ce type de dispositif, qui repose sur la mise en lien d'œuvres littéraires, en décrivant d'abord différents types de réseaux, en présentant ensuite la manière dont un enseignant planifie son année scolaire en organisant les tâches confiées aux élèves autour de réseaux variés et, enfin, en mettant en lumière divers obstacles à leur création ainsi que des pistes pour aider les enseignants à les surmonter.

\section{Using Literary Networks to Integrate Children's Literature into the Classroom}

Literary networks are very helpful when integrating children's literature in the development of language and subject competencies of students. In this article, we will begin the exploration of literary works by describing the different types of networks, followed by suggestions to help the teacher plan the school year with tasks for the students centred on these networks. We conclude by highlighting the obstacles to their creation as well as solutions to help the teachers overcome them.

\section{Cómo integrar la literatura juvenil en clase} a través de las redes literarias

Para integrar la literatura juvenil en clase y respaldar las competencias lingüísticas y disciplinarias de los alumnos, las redes literarias constituyen uno de los recursos más valiosos. En este artículo, exploramos este tipo de dispositivo, que consiste en poner en línea las obras literarias, describiendo distintos tipos de redes, presentando el método de planificación escolar anual de un profesor que organiza las tareas que adjudica a los alumnos en torno a diversas redes y, por último, destacando los obstáculos que impiden su creación, así como los lineamientos necesarios para ayudar a los profesores a superarlos.
$\mathrm{P}$ our plusieurs pays de la francophonie, la littérature jeunesse semble occuper une place grandissante dans les écoles (Devanne 2006a; Morin \& Montésinos-Gelet 2007). Au Québec, avec l'arrivée de la compétence "Apprécier des œuvres littéraires" au sein du Programme de formation de l'école québécoise (Ministère de l'Éducation, du Loisir et du Sport (MELS) 2001) et la création en 2002 du site Internet Livres ouverts (<www.livresouverts.qc.ca $>$ ), les directives ministérielles ont probablement contribué à ce développement de l'intérêt pour l'univers de la littérature jeunesse. Au-delà de la lecture plaisir, de plus en plus d'enseignants se tournent donc vers la littérature jeunesse à des fins d'enseignement. L'offre grandissante d'ouvrages didactiques (voir notamment Duprey \& Duprey 2006; Morin \& Montésinos-Gelet 2007; Nadon 2007; Nadon 2011; Schneider 2008) et de revues (p. ex. Le Pollen) sur le sujet témoigne d'ailleurs clairement de cet intérêt. Plusieurs recherches ont documenté l'usage des œuvres de littérature jeunesse comme outil didactique pour l'enseignement des compétences en français. Celles-ci tendent à montrer qu'être en contact avec des œuvres de qualité amène les élèves à développer leurs compétences en français (Morin \& Montésinos-Gelet 2007), leur motivation à lire des œuvres diversifiées et à écrire (Dupin de Saint-André \& Montésinos-Gelet 2009). Par contre, cette intégration des œuvres de littérature jeunesse en classe n'est pas aisée pour tous, différents obstacles sont relevés par les enseignants. En effet, intégrer la littérature jeunesse en classe demande de se repérer dans l'univers foisonnant de la littérature jeunesse et surtout de savoir de quelle façon présenter les œuvres aux élèves pour leur permettre de développer les compétences du Programme de formation de l'école québécoise (MELS 2001).

Les réseaux littéraires constituent un dispositif très intéressant pour l'intégration des œuvres littéraires en classe ainsi qu'un moyen d'amener les élèves à établir des liens entre les œuvres, comme recommandé dans la Progression des apprentissages au primaire en français (MELS 2009). Dans cet article, nous explorons ce type de dispositif en fonction de quatre axes : une description des réseaux, une étude de cas d'une planification annuelle organisée autour de réseaux variés, une mise en lumière des obstacles à la création de réseaux et des besoins des enseignants quant à celle-ci et des sugges- 
tions quant au rôle du bibliothécaire pour l'intégration de réseaux littéraires en classe.

\section{Les réseaux littéraires}

Un réseau littéraire est un regroupement de livres organisé en fonction d'un questionnement qui peut porter sur différents aspects (p. ex. un auteur ou un personnage) (Boutevin \& Richard-Principalli 2008). Ce regroupement est conçu en vue d'éclairer ce questionnement sous différents angles, en mettant ces œuvres "en résonance» (Boutevin \& Richard-Principalli 2008, 226). "Les textes sont agencés de manière à pouvoir se relancer, se compléter et permettre aux élèves d'approfondir leur compréhension, mais aussi leur interprétation et leur appréciation. » (Morin \& Roger 2014, 95) Ainsi, la réponse au questionnement vient des liens que les lecteurs établissent entre les œuvres du réseau. Ce réseau sera le point de départ d'activités de lecture, d'écriture et, parfois, d'intégration des matières qui viseront à répondre au questionnement initial en amenant les lecteurs à établir des liens entre les différentes œuvres.

\section{Les différents types de réseaux littéraires}

Catherine Tauveron est une des chercheuses qui s'est grandement intéressée à l'utilisation des réseaux littéraires en classe. En 2002, elle en a proposé une typologie. Au sein de celle-ci, nous avons sélectionné les réseaux qui nous semblent les plus intéressants pour une exploitation en classe en fonction du Programme de formation de l'école québécoise (MELS 2001). Certains types de réseaux ont également été ajoutés à la typologie de cette chercheuse (voir le Tableau 1 pour une présentation des types de réseaux).
Les types de réseaux peuvent aussi se chevaucher. Ainsi, étudier les œuvres de Chris Van Allsburg constitue à la fois un réseau d'auteur et un réseau autour du genre fantastique. Des exemples des différents réseaux sont proposés dans la partie suivante pour étayer la pertinence d'intégrer ce type de dispositif en classe.

\section{Pertinence de la mise en œuvre de réseaux littéraires en classe}

Une des problématiques fréquemment soulevées par les enseignants, quand ils commencent à intégrer la littérature jeunesse en classe, consiste à ne pas savoir comment organiser leur enseignement autour des livres de façon à relier entre elles les différentes activités pour créer un tout cohérent. Opter pour des réseaux littéraires peut être une porte d'entrée rassurante pour les enseignants en leur permettant de faire un choix de livres éclairé à mettre en relation avec une intention pédagogique.

Tauveron (2004) relève quatre grandes fonctions des réseaux littéraires : 1) développer chez les élèves l'habitude de mettre en relation les textes; 2) leur permettre de " construire et de structurer» leur culture; 3 ) les amener à comprendre et à interpréter des textes en prenant appui sur cette culture, et finalement; 4) leur donner la possibilité, " en tant que dispositif multipliant les voies d'accès au texte, d'y pénétrer avec plus de finesse, d'y découvrir des territoires autrement inaccessibles, d'éclairer des zones autrement laissées dans la pénombre " (Tauveron 2004, 72). À ces fonctions, nous pouvons d'une part ajouter que les réseaux littéraires offrent aussi aux enseignants la possibilité de structurer leur enseignement pour que la lecture, l'écriture et l'oral y soient interreliés et, d'autre part, qu'ils constituent d'excellentes occa-

\section{Tableau 1}

Différents types de réseaux littéraires (adaptés à partir de Tauveron 2002, 206-207)1

\begin{tabular}{|c|c|}
\hline Types de réseaux & Définitions \\
\hline Réseau autour d'un thème unificateur & Choix d'œuvres autour d'un thème dans le but de répondre à une intention pédagogique \\
\hline Réseau d'auteur & Choix d'œuvres d'un auteur afin de dégager les particularités de son style et de son univers \\
\hline Réseau d'illustrateur & Choix d'œuvres d'un même illustrateur afin de dégager les caractéristiques de son style \\
\hline Réseau autour d'un personnage stéréotype & $\begin{array}{l}\text { Choix d'œuvres pour mieux connaître un personnage stéréotype de la littérature jeunesse } \\
\text { (p. ex. le loup, l'ogre, la sorcière ou le superhéros) }\end{array}$ \\
\hline Réseau générique & Choix d'œuvres appartenant au même genre littéraire \\
\hline Réseau hypertextuel & Choix d'œuvres se rapportant à un texte source (p. ex. un conte et ses adaptations) \\
\hline Réseau intertextuel & $\begin{array}{l}\text { Choix d'une œuvre dans laquelle il y a de l'intertextualité (références à d'autres textes) } \\
\text { et présentation des autres œuvres auxquelles elle se rapporte }\end{array}$ \\
\hline Réseau autour d'un procédé d'écriture & Choix d'œuvres dans lesquelles le ou les auteurs utilisent un même procédé d'écriture \\
\hline
\end{tabular}

1. La typologie initiale de Tauveron (2002) comprend d'autres réseaux (p. ex. autour des mythes et des légendes et autour des symboles) qui, selon nous, peuvent être compris au sein d'autres réseaux de cette catégorisation. De plus, à la typologie initiale, nous avons ajouté le réseau sur le travail d'un illustrateur, particulièrement pertinent pour intégrer l'enseignement des arts en classe. 
sions d'intégrer les différentes matières (p. ex. éthique, arts, univers social, mathématique, science). Comme chaque type de réseau possède une utilité différente pour une exploitation en classe, il est primordial de diversifier ces choix en ce qui a trait aux réseaux.

\section{Le réseau autour d'un thème unificateur}

Le réseau autour d'un thème unificateur est le candidat idéal pour une intégration de différentes matières. Par exemple, un réseau autour de la thématique des sociétés non démocratiques, composé des albums Mao et moi, Le mur, Le piano rouge et La rédaction, permettrait à la fois de cibler des compétences en français et en univers social en lien avec le Programme de formation de l'école québécoise (MELS 2001) pour le $3^{\mathrm{e}}$ cycle du primaire. Dans un autre registre, la pertinence de ce type de réseau a été mise à plusieurs reprises de l'avant concernant l'enseignement de l'éthique (Dupin de Saint-André \& Montésinos-Gelet 2012) ou de la philosophie pour enfants (Chirouter 2007).

\section{Le réseau d'auteur}

Le réseau sur un auteur est très pertinent pour amener les élèves à écrire à partir des œuvres de littérature jeunesse. En effet, étudier en profondeur le style d'un auteur, à l'aide par exemple des six traits d'écriture ${ }^{1}$, est une voie à privilégier pour aider le processus d'écriture. Ce type de réseau permet aussi bien souvent d'avoir une compréhension approfondie de l'univers d'un auteur, car " un véritable auteur de littérature de jeunesse compose un monde imaginaire qui lui est personnel et au fil des années élabore une cuvre " (Devanne 2006b, 117). Pensons par exemple à l'auteur Geoffroy de Pennart qui dans chacune de ses ouvres convoque des personnages de ses propres œuvres, leur donnant tour à tour des rôles principaux, puis secondaires, créant ainsi un univers où tout s'entrecoupe. Enfin, un réseau d'auteur peut aussi donner la possibilité aux élèves d'interpréter plus finement une des œuvres de Geoffroy de Pennart en s'appuyant sur leurs connaissances de ses autres livres. Ainsi, connaître l'univers de de Pennart et les caractéristiques d'Igor, un de ses personnages fétiches, conduiront sûrement les élèves à mieux interpréter la fin d'Igor et les trois petits cochons du même auteur à la lumière de ces connaissances.

\section{Le réseau d'illustrateur}

Un réseau d'illustrateur est une façon très intéressante d'intégrer les arts à l'enseignement du français. C'est aussi la possibilité de faire remarquer aux élèves que, à l'instar de l'auteur, l'illustrateur a une intention qui influera sur ses choix : le contenu de son illustration,

1. Pour une description de ces traits, voir Olness (2005). son cadrage, sa technique, ses matériaux et ses couleurs, le rapport texte-image, la place de l'illustration sur la double page et le montage des illustrations. Plonger dans l'univers de différents illustrateurs, c'est donner à voir aux élèves des mondes très différents dont ils pourront s'inspirer en art plastique.

\section{Le réseau autour d'un genre}

Un réseau sur un genre donne la possibilité aux élèves d'étudier les caractéristiques de celui-ci, mais aussi de constater de quelle façon les auteurs les transgressent pour créer une œuvre originale (Tauveron 2002). À titre d'exemple, étudier différents albums sur le genre policier permettra aux élèves de saisir que Bénédicte Guettier le parodie dans sa série des enquêtes de l'inspecteur Lapou.

\section{Le réseau hypertextuel}

Un réseau hypertextuel est, quant à lui, l'occasion idéale pour faire prendre conscience aux élèves de l'originalité de la production littéraire actuelle. Songeons au conte. Partir d'un conte source, le relier à ses adaptations et à des contes détournés est idéal pour que les élèves constatent qu'une même histoire peut se décliner de multiples façons.

\section{Le réseau intertextuel}

Tout comme le réseau hypertextuel, le réseau intertextuel sera un moyen à privilégier pour faire constater aux élèves qu'au sein du monde littéraire, les auteurs et les illustrateurs aiment convoquer d'autres textes dans leurs œuvres. Ces textes peuvent être les leurs ou ceux d'auteurs connus. Créer ce type de réseau, c'est participer au développement de la culture littéraire et agir en tant que passeur culturel en sensibilisant les élèves aux liens étroits qui existent entre différents textes. À titre d'exemple, la mise en relation de Mon ballon de Mario Ramos avec ses œuvres précédentes, qu'il convoque dans son album, fournira des éléments qui, sans cette mise en relation, resteraient inaccessibles pour les élèves.

\section{Le réseau centré sur un procédé d'écriture}

Le réseau centré sur un procédé d'écriture est, comme celui centré sur un auteur, très propice à la mise en place d'une situation d'écriture avec les élèves. En effet, il vise à découvrir le type de procédé employé par les auteurs et à l'analyser pour en comprendre le fonctionnement. À ce sujet, la série "Chester » de Mélanie Watt pourrait être étudiée pour dégager les caractéristiques de la métanarration qui s'y retrouve.

En somme, comme chaque type de réseau présente des avantages différents pour une mise en œuvre en classe, l'enseignant doit planifier chaque réseau avec soin, mais aussi penser l'arrimage entre les différents 
réseaux et diversifier les types de réseaux au sein d'une année scolaire.

\section{Étude de cas d'une planification annuelle centrée sur des réseaux variés}

De manière à dégager les aspects à considérer pour soutenir des enseignants qui souhaitent adopter les réseaux littéraires en classe, nous avons choisi de présenter la manière dont un enseignant expert s'y prend.

Après une brève présentation de ses caractéristiques comme enseignant et du contexte actuel de sa pratique, il est donc question dans cette étude de cas de la manière dont Robert s'y prend pour construire une planification annuelle de réseaux, des œuvres qu'il sélectionne et des liens qu'il établit entre les différents réseaux exploités.

Robert est un enseignant qui peut être qualifié d'expert. Exerçant depuis près de 30 ans dans les différents cycles du primaire, il termine actuellement une maîtrise en didactique du français, il a participé à de nombreux projets de recherche et il a été conseiller pédagogique en français. Ses connaissances des œuvres littéraires sont exceptionnelles et ses pratiques d'enseignement correspondent aux critères définis par Hall (2003) pour distinguer les enseignants les plus efficaces.
Lors de l'année scolaire 2013-2014, Robert a enseigné dans une classe d'adaptation scolaire (Groupe adapté de développement cognitif, social et affectif) accueillant des enfants de six ans ayant fréquenté une classe préscolaire cinq ans, mais qui ne répondaient pas aux attentes du Programme de formation de l'école québécoise (MELS 2001) préscolaire en raison d'un manque d'autonomie, de difficultés affectives et sociales, et de difficultés dans l'acquisition des habiletés langagières et motrices. Son mandat, dans le cadre de ce projet pilote chapeauté par la Commission scolaire de la Vallée-desTisserands et le syndicat des enseignants, était de stimuler les enfants en fonction de leurs besoins particuliers pour favoriser la poursuite de leur scolarité en milieu régulier, de développer des habiletés favorisant l'autonomie, la concentration, la mémoire, les habiletés sociales et la motricité et de consolider les apprentissages du programme préscolaire en introduisant progressivement celui de la $1^{\text {re }}$ année afin d'outiller les élèves pour leur passage au primaire. Pour répondre à ce mandat, Robert a choisi de recourir à des réseaux littéraires variés.

\section{La planification annuelle de Robert}

Le Tableau 2 ci-dessous présente la planification annuelle de Robert. Au fil de l'année scolaire, il a exploité

\section{Tableau 2}

Planification annuelle de Robert

\begin{tabular}{|c|c|c|c|c|}
\hline Quand? & Titre du réseau & Intention & Nature du réseau & Finalité \\
\hline Septembre/octobre & Les superhéros & $\begin{array}{l}\text { - Créer des liens avec les élèves } \\
\text { - Établir une correspondance } \\
\text { entre le personnage et nous } \\
\text { - Découvrir le monde des superhéros }\end{array}$ & $\begin{array}{l}\text { Autour d'un personnage } \\
\text { stéréotype }\end{array}$ & $\begin{array}{l}\text { - Accomplir la mission de Transito- } \\
\text { nique (chasse au trésor) } \\
\text { - Devenir un superhéros } \\
\text { (parade de mode) }\end{array}$ \\
\hline Octobre/novembre & $\begin{array}{l}\text { Les sorcières } \\
\text { (personnage stéréotype) }\end{array}$ & $\begin{array}{l}\text { - Découvrir le monde des sorcières } \\
\text { - Travailler la description } \\
\text { du personnage }\end{array}$ & $\begin{array}{l}\text { Autour d'un personnage } \\
\text { stéréotype }\end{array}$ & $\begin{array}{l}\text { - Créer un sorcier ou une sorcière } \\
\text { et en faire la description } \\
\text { - Créer un catalogue de sorciers } \\
\text { et de sorcières }\end{array}$ \\
\hline Novembre/décembre & Éric Carle & - Découvrir un auteur et son univers & Autour d'un auteur-illustrateur & - Écrire à la manière de... \\
\hline Décembre & Noël & - Découvrir Noël à travers les albums & Autour d'un thème unificateur & - S'imprégner de l'esprit de Noël \\
\hline Janvier & La correspondance & $\begin{array}{l}\text { - Travailler les différents types } \\
\text { de correspondance }\end{array}$ & Autour d'un procédé d'écriture & $\begin{array}{l}\text { - Écrire à notre superhéros } \\
\text { (membre de la famille, ami, etc.) }\end{array}$ \\
\hline Février/mars & La moufle & $\begin{array}{l}\text { - Travailler la structure répétitive } \\
\text { par accumulation } \\
\text { - Comparer différentes versions } \\
\text { du conte et des adaptations }\end{array}$ & $\begin{array}{l}\text { Réseau hypertextuel et autour } \\
\text { d'un procédé d'écriture }\end{array}$ & $\begin{array}{l}\text { - Écrire un conte collectif } \\
\text { à la manière de la moufle } \\
\text { - Écrire un petit livre: } \\
\text { « Dans ma moufle, il y a... » }\end{array}$ \\
\hline Mars & Le loup & $\begin{array}{l}\text { - Le loup est-il aussi méchant } \\
\text { qu'on le prétend? }\end{array}$ & $\begin{array}{l}\text { Autour d'un personnage } \\
\text { stéréotype }\end{array}$ & $\begin{array}{l}\text { - Tirer une conclusion sur le loup. } \\
\text { Est-il méchant ou gentil? }\end{array}$ \\
\hline Avril/mai & $\begin{array}{l}\text { Augustine } \\
\text { et ses peintres }\end{array}$ & $\begin{array}{l}\text { - Découvrir des peintres } \\
\text { qui ont marqué l'histoire }\end{array}$ & $\begin{array}{l}\text { Autour d'un thème unificateur } \\
\text { (les arts) }\end{array}$ & $\begin{array}{l}\text { - Créer un musée } \\
\text { Exposition des œuvres réalisées } \\
\text { par les élèves inspirées des } \\
\text { différents peintres étudiés }\end{array}$ \\
\hline Mai/juin & $\begin{array}{l}\text { Les fonds marins } \\
\text { poissons }\end{array}$ & $\begin{array}{l}\text { - Connaître le monde marin et } \\
\text { les différentes espèces qui y vivent }\end{array}$ & Autour d'un thème unificateur & - Créer un fonds marin \\
\hline
\end{tabular}


Figure 1

Planification des activités du réseau sur les sorcières

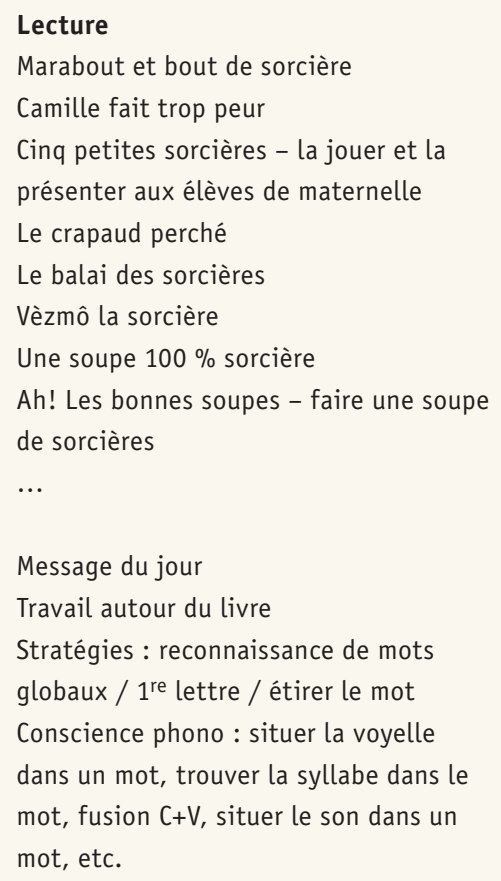

\section{Mathématique}

Nombres 0 à 10 - comptine

Nombres 11 à 16 - nom

Lire, nommer et reconnaître les nombres

Plus grand, plus petit

Jeu de l'arbre (0 à 16) :

compter, dénombrer, placer

\section{Intention :}

Découvrir le monde des sorcières.

Travailler la description du personnage.

Axe :

Autour d'un personnage stéréotype

Finalité :

Créer un sorcier ou une sorcière et en faire la description.

Créer un catalogue de sorciers

et de sorcières.

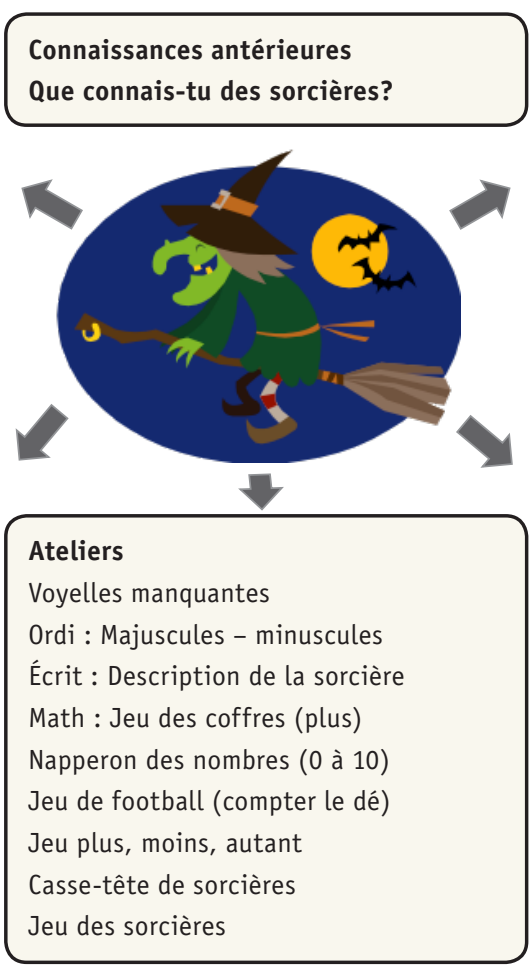

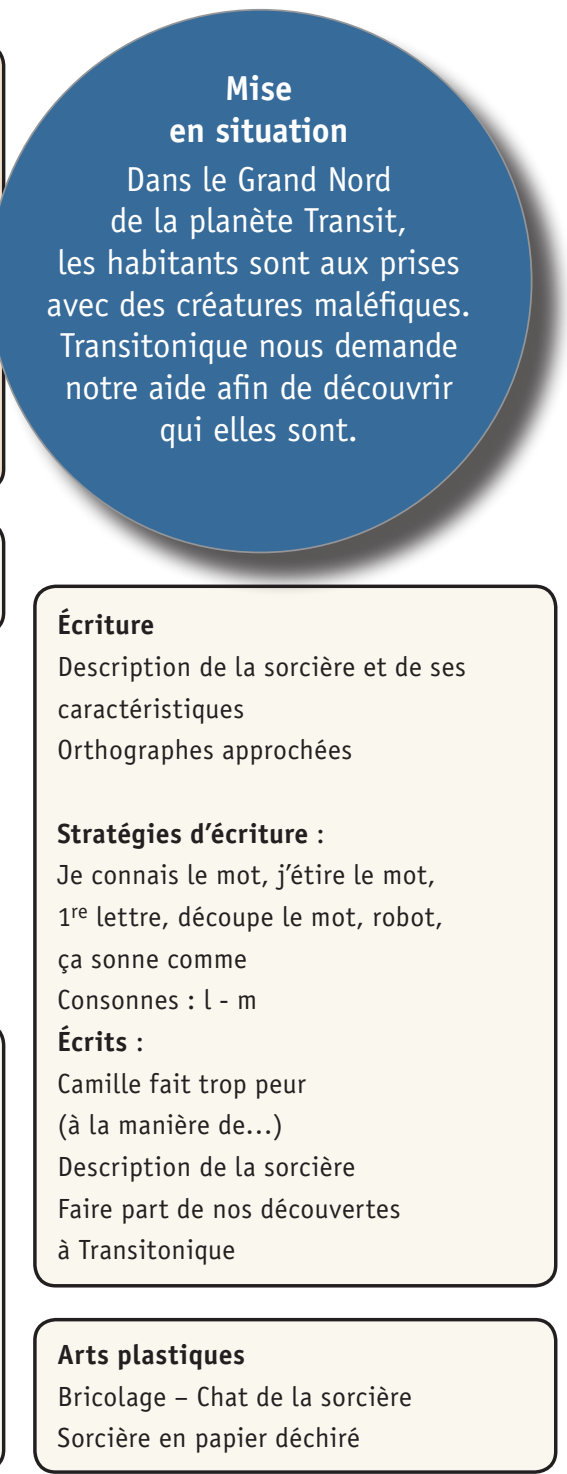

avec ses élèves neuf réseaux différents. Parmi ceux-ci, trois concernaient un personnage stéréotype (les superhéros, les sorcières, le loup), trois relevaient d'un thème unificateur (Noël, les arts, les fonds marins), un présentait un auteur-illustrateur (Eric Carle) et un autre, un réseau hypertextuel autour du conte La moufle, permettait d'aborder la structure répétitive accumulative. Un second procédé d'écriture, la correspondance, a également fait l'objet d'un réseau. La durée de l'exploitation des réseaux est variable, allant de deux semaines (sur le thème de Noël) à un mois et demi. Toutes les activités de classe sont reliées au réseau. Comme l'objectif de cette étude de cas n'est pas de mettre en relief la créativité et l'excellence didactique de Robert, nous ne détaillerons pas ses planifications d'activités pour chacun des réseaux, mais la Figure 1 illustre sommairement la manière dont il s'y prend.
Robert a des raisons variées lorsqu'il choisit les réseaux littéraires qu'il va explorer avec ses élèves. Comme beaucoup d'enseignants, les fêtes qui ponctuent l'année scolaire orientent quelques-uns de ses choix (les sorcières lors de l'Halloween, le thème de Noël en décembre). Il reprend parfois certains réseaux d'une année à l'autre, mais il apprécie également en développer de nouveaux. Il a déjà mis en œuvre au début d'autres années le réseau sur les superhéros qu'il juge très pertinent pour créer des liens avec ses élèves, établir les règles de vie de la classe et mettre les élèves en confiance quant à leur pouvoir d'apprendre. Il affectionne également les réseaux hypertextuels autour de contes classiques. Il a toutefois tendance à changer régulièrement de conte d'une année à l'autre. Il procède de même pour les réseaux d'auteur ou d'illustrateur. Il en prévoit toujours au moins un, mais son choix varie. 


\section{Le choix des œuvres}

Passionné de littérature de jeunesse depuis des années, Robert a fortement investi dans l'achat d'œuvres de qualité. Il fréquente assidument les bibliothèques et les libraires pour découvrir de nouvelles œuvres, il reçoit avec intérêt les conseils de lecture des professionnels qu'il y côtoie de même que ceux d'autres enseignants aussi engagés que lui, et il a recours à des ressources comme Livres ouverts, Lurelu, Le pollen, La revue des livres pour enfants et Ricochet pour orienter ses choix.
Lorsque Robert exploite un réseau, les élèves ont accès à une très grande quantité d'œuvres, certaines sont abordées collectivement par des lectures interactives ou par des activités d'écriture, alors que d'autres sont disponibles pour des lectures libres qui alimentent l'intérêt des élèves envers le réseau travaillé. Au début de l'exploitation d'un réseau, Robert présente l'ensemble des œuvres aux élèves de façon à susciter leur curiosité et à les inviter à découvrir en lecture libre celles qui ne seront pas exploitées en classe.

Pour illustrer sa manière de faire, le choix des œuvres du réseau à propos de l'auteur-illustrateur Éric Carle est présenté dans le Tableau 3.

\section{Tableau 3}

Les œuvres d'Éric Carle exploitées collectivement par Robert et ses élèves

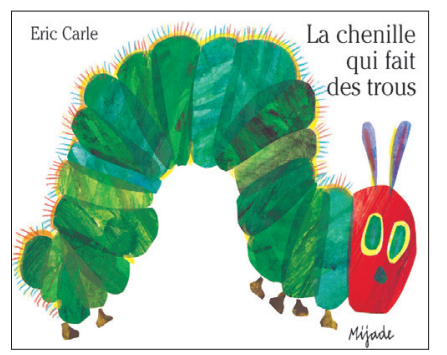

- Lecture interactive

- Mise en scène à l'aide de marottes pour les élèves de maternelle

- Arts plastiques : création de chenilles en gouache, en papier découpé, au pastel

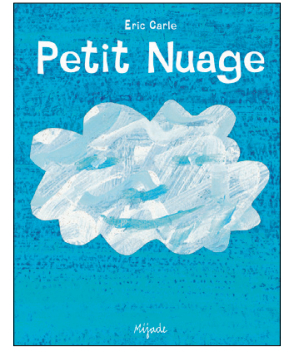

- Lecture interactive

Écriture à la manière de Petit Nuage
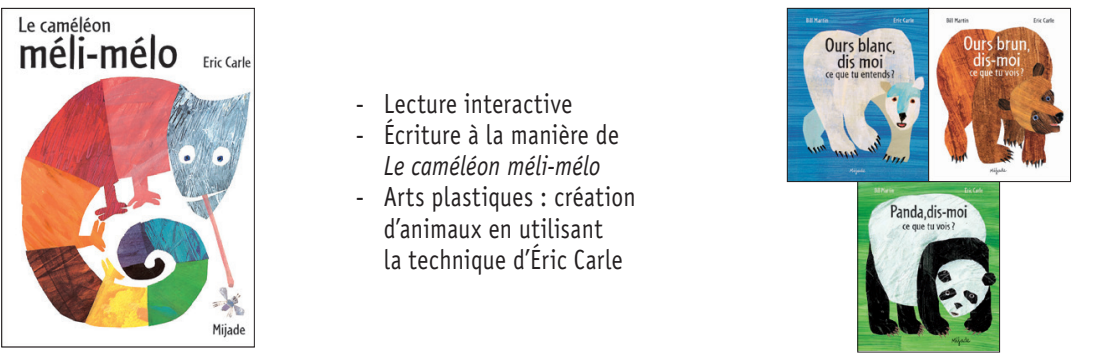

Lecture interactive

- Écriture à la manière de Le caméléon méli-mélo

- Arts plastiques : création

d'animaux en utilisant

la technique d'Éric Carle

Écriture à la manière de Ours blanc, dis-moi

Arts plastiques : création d'animaux en utilisant la technique d'Eric Carle

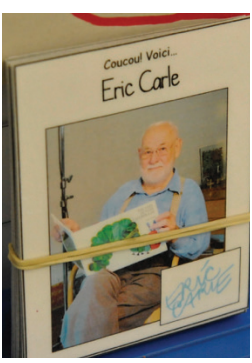

À partir d'informations puisées sur Internet, Robert crée des fiches informatives sur tous les auteurs qu'il aborde dans le cadre d'un réseau. Il le fait également lorsqu'il lit plusieurs oeuvres d'un auteur dans le cadre d'un autre réseau (p. ex. Élise Gravel, pour sa série « Les super machins » dans le cadre du réseau sur les superhéros).

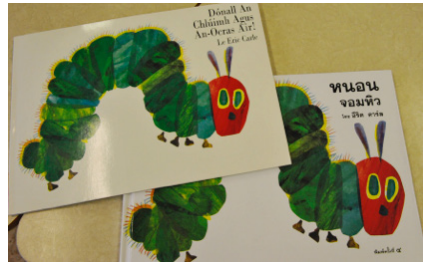

Lorsqu'il trouve des œuvres qu'il exploite avec ses élèves dans des éditions en langues étrangères,

il leur donne accès afin d'éveiller leur conscience à la variété des langues et des systèmes d'écriture. 
Robert a choisi l'ordre de présentation des œuvres. Il a commencé par La chenille qui fait des trous, l'œuvre la plus célèbre d'Éric Carle. Il a poursuivi avec deux autres albums de cet auteur et illustrateur. Puis, il a présenté la série « Dis-moi », réalisée en collaboration avec Bill Martin.

Les œuvres travaillées collectivement ont été lues plusieurs fois chacune. Les autres œuvres de ce remarquable auteur-illustrateur étaient à la disposition des élèves pour des lectures et des explorations libres.

\section{Les liens tissés entre les réseaux}

Un aspect particulièrement intéressant du travail en réseaux de Robert est la manière dont il construit des transitions entre les réseaux. En voici quelques exemples.

L'introduction du réseau relatif aux sorcières impliquait Transitonique, le superhéros mis en scène dans le réseau précédent. La mise en situation proposée aux élèves était formulée en ces termes : «Dans le Grand Nord de la planète Transit, les habitants sont aux prises avec des créatures maléfiques. Transitonique nous demande notre aide afin de découvrir qui elles sont. »

Par ailleurs, la dernière œuvre présentée aux élèves dans le cadre du réseau sur le thème de Noël était Le facteur du Père Noël d'Allan et Janet Ahlberg, elle constituait une transition vers le réseau relatif à la correspondance. Lors du travail sur ce réseau, les élèves écrivaient régulièrement à Transitonique, le superhéros de la classe.

\section{Quelques aspects à considérer dans la planification de réseaux en classe}

Au regard de cette étude de cas, il est possible de dégager divers aspects à considérer pour soutenir des enseignants qui souhaitent adopter des réseaux littéraires en classe :

- choisir des réseaux de nature variée;

- identifier le moment de l'année le plus pertinent pour les exploiter en fonction de ce qu'ils permettent de mettre en œuvre avec les élèves;

- anticiper la durée de l'exploitation des réseaux en fonction des projets qui y sont associés;

- sélectionner les œuvres à travailler collectivement et les œuvres complémentaires;

- réfléchir à l'ordre de présentation des œuvres aux élèves;

- faire des liens entre les différents réseaux.

\section{Obstacles à la mise en œuvre de réseaux en classe et des besoins des enseignants quant au choix des œuvres}

$\mathrm{Au}$ premier abord, construire un réseau littéraire peut paraître relativement simple, mais c'est en s'attelant à cette tâche que bien souvent les enseignants constatent sa complexité.

Pour commencer, il faut sélectionner le type de réseau qui sera exploité ainsi que l'intention pédagogique qui sera au cœur du réseau. Pour ce faire, il est nécessaire de connaître les différents types de réseaux ainsi que ce qu'ils permettent de travailler avec les élèves. Puis, il faut choisir les œuvres qui seront au cœur du réseau, ce qui demande une véritable réflexion. À titre d'exemple, les classes de maternelle ou de $1^{\text {er }}$ cycle fonctionnent bien souvent autour d'une approche thématique. Or, le réseau autour d'un thème unificateur est quelque peu différent de ce type d'approche. Pour le mettre en œuvre, il ne suffit pas de recueillir un certain nombre d'œuvres qui touchent une même thématique. Il faut, bien au contraire, sélectionner les œuvres avec beaucoup d'attention de façon à éclairer chaque aspect de cette thématique, ce qui est compliqué en raison de la profusion d'œuvres pour la jeunesse disponibles tant en bibliothèque qu'en librairie. Imaginons que nous souhaitions créer un réseau sur le thème de l'identité pour le $1^{\text {er }}$ cycle, en ayant comme intention pédagogique de réfléchir au caractère unique de chaque être humain en lien avec le programme d'éthique pour ce cycle (MELS 2007). Une recherche sur le site Livres ouverts du MELS combinant les deux critères "identité " et " $1^{\text {er }}$ cycle " permet de constater que de nombreux albums de qualité destinés à des élèves de ce niveau traitent de cette thématique, comme en témoignent les 56 résultats obtenus. Comment alors choisir parmi ceux-ci ceux qui seront au cœur du réseau littéraire? Il devient alors primordial de sélectionner des œuvres pour aborder cette thématique selon différents angles en fonction de l'intention pédagogique. De plus, il faudra veiller à effectuer ce choix en pensant aux dialogues à établir entre les différentes œuvres. Un tel réseau pourrait donc comporter les trois axes suivants : moi (« qui suis-je?»), moi vu par les autres (" qui suis-je pour l'autre?») et devenir un autre ("faut-il que je change? »). Les œuvres seront alors choisies en fonction de ces axes ${ }^{2}$. C'est lors de cette étape déterminante que les bibliothécaires pourront être d'un grand secours pour soutenir les enseignants dans leur sélection d'œuvres de qualité.

L'étape ultérieure consiste à organiser les œuvres entre elles, à savoir quelle progression sera envisagée en fonction de l'intention pédagogique initiale. Là encore, les bibliothécaires pourront faire des suggestions aux

2. Voir Le pollen (no 2) pour des idées d'œuvres tournant autour de ces trois axes. 
enseignants quant à leur agencement en fonction notamment de leur complexité ou bien encore, pour les contes, de leur ordre initial de publication.

Pour chacune des œuvres, l'enseignant devra par la suite opter pour un dispositif en lecture ou en écriture adapté aux caractéristiques de l'œuvre. Ainsi, une œuvre résistante, qui demande un travail pour être comprise ou interprétée, se prêtera bien à une lecture interactive, tandis qu'une œuvre plus simple pourra simplement être lue à haute voix pour que les élèves puissent la découvrir.

Pour finir, Tauveron (2002) conseille que l'enseignant choisisse des œuvres et des documentaires qui, sans être au centre du réseau, graviteront autour de celui-ci pour enrichir la culture littéraire commune des élèves. Ceux-ci pourront s'appuyer sur ces livres pour aborder les œuvres du réseau et ainsi tisser des liens entre elles. Ces œuvres peuvent porter sur une thématique similaire ou bien permettre de découvrir d'autres œuvres d'un auteur étudié dans le réseau. Pour ce faire, l'aide des bibliothécaires leur sera encore précieuse pour veiller à présenter une grande variété de livres aux élèves en lien avec ce réseau.

Lorsqu'il est question d'apporter des changements pour intégrer la littérature jeunesse en classe, un des obstacles majeurs relevés par les enseignants est d'ordre matériel. Lors de la création de réseaux littéraires, l'accessibilité aux livres qui les composent peut être problématique dans certains milieux scolaires.

En lien avec ces obstacles, nous proposons, dans la dernière partie, des suggestions quant au rôle que les bibliothécaires scolaires peuvent jouer dans la création de réseaux littéraires.

\section{Suggestions quant au rôle des bibliothécaires scolaires pour l'implantation de réseaux littéraires au sein des écoles}

Avec la mise en œuvre du Plan de lecture à l'école du MELS, chaque commission scolaire devait embaucher au moins un bibliothécaire (Fédération des professionnelles et professionnels de l'éducation du Québec (FPPE-CSQ) 2013). Même si certaines commissions scolaires ne comptent toujours pas de bibliothécaire scolaire, le nombre de ces professionnels au sein des commissions scolaires a considérablement augmenté au cours des six dernières années (FPPE-CSQ 2013). Cependant, il semblerait que les rôles qui leur sont attribués varient grandement d'une commission scolaire à l'autre (Baillargeon, Charest, Fortin \& Moreau 2014; FPPE-CSQ 2013).

Dans cet article, de façon à nous questionner sur le rôle des bibliothécaires dans la création des réseaux littéraires, nous avons choisi de nous baser sur les mandats généraux de cette profession relevés par Moreau (2013) (voir Tableau 4).

Tous les mandats mis en lumière par Moreau (2013) ne s'appliquent pas à l'implantation de réseaux littéraires au sein des écoles, mais plusieurs d'entre eux nous semblent très pertinents.

Premièrement, les bibliothécaires scolaires pourraient former et accompagner les enseignants dans le choix des ressources qu'ils peuvent utiliser pour sélectionner des œuvres de qualité et les organiser au sein d'un réseau littéraire. En guise de ressources, nous pouvons notamment penser au site Livres ouverts ${ }^{3}$ ainsi

Tableau 4

Mandats généraux de la profession de bibliothécaire scolaire (Moreau 2013, 116)

1. Soutenir les apprentissages des élèves par des formations et accompagnements proposés aux enseignants :

- compétences informationnelles, pour institutionnaliser une didactique de la recherche d'informations et développer l'esprit critique des élèves;

- littérature jeunesse, pour soutenir leur rôle de passeur culturel auprès des élèves;

- utilisation maximale de la bibliothèque scolaire, pour changer les pratiques actuelles et faire de la bibliothèque un prolongement de la classe.

2. Restructur[er] et réaménag [er] les bibliothèques scolaires, primaires et secondaires, pour en faire des centres d'apprentissages pédagogiques, culturels, numériques et collaboratifs.

3. Développ [er] les collections des bibliothèques, incluant l'évaluation et l'élagage.

4. Travaill[er] en collaboration avec les autres professionnels de la CS [commission scolaire] pour assurer la cohérence des accompagnements dans les écoles en ce qui concerne les compétences à lire des élèves.

5. Offr[ir] un service de veille, notamment pour les professionnels et les cadres des CS.

6. Milit[er] pour la création d'une plateforme virtuelle, actuellement inexistante, qui permettra l'achat, le stockage et le prêt de livres numériques pour les élèves et les enseignants [...].

3. Toute une section est consacrée aux réseaux littéraires sur le site Livres ouverts <www.livresouverts.qc.ca/index.php?p=dp\&ss=reseaux_livres $>$. 
qu'à d'autres sites Internet spécialisés en littérature jeunesse (p. ex. Ricochet et Télémaque) ou bien à des revues spécialisées dans ce domaine (Le pollen, Lurelu, La revue des livres pour enfants). Se perfectionner quant à l'utilisation de ces différents outils permettrait aux enseignants de se repérer plus facilement dans l'univers foisonnant de la littérature jeunesse. De plus, en collaboration avec les conseillers pédagogiques, les bibliothécaires scolaires pourraient accompagner les enseignants pour la constitution et l'organisation de ces réseaux littéraires en lien avec le Programme de formation de l'école québécoise (MELS 2001).

Deuxièmement, enseignants et bibliothécaires scolaires pourraient allier leur expertise pour faire en sorte que les réseaux littéraires vécus en classe trouvent un prolongement au sein de la bibliothèque de l'école. Ce prolongement pourrait prendre différentes formes: mise en valeur d'œuvres en lien avec le réseau travaillé en classe, animation autour de celui-ci, diffusion des créations des élèves au sein de la bibliothèque, etc.

Troisièmement, si le désir d'implanter des réseaux littéraires est présent au sein d'une école, il serait particulièrement intéressant que la collection de la bibliothèque scolaire puisse être développée en fonction de certains choix de réseaux littéraires. D’une part, les bibliothécaires pourraient proposer aux enseignants des réseaux qu'ils pourraient emprunter pour un usage en classe et, d'autre part, ils pourraient offrir aux élèves des œuvres complémentaires à ces réseaux. Pour les commissions scolaires qui se dotent d'un centre de ressources pédagogiques, comme décrit par Baillargeon, Charest, Fortin et Moreau (2014), il pourrait être envisageable d'y centraliser un grand nombre de réseaux littéraires que les enseignants pourraient emprunter pour une exploitation en classe.

Quatrièmement, de façon à promouvoir l'usage des réseaux littéraires, les bibliothécaires pourraient travailler de concert avec les conseillers pédagogiques afin de former les enseignants à la planification et à l'exploitation des réseaux en classe en lien avec le Programme de formation de l'école québécoise (MELS 2011). Une telle collaboration a notamment trouvé sa place au sein de la Commission scolaire des Bois-Francs.

Enfin, si une plateforme virtuelle est développée de façon à permettre le prêt de livres numériques au sein des bibliothèques scolaires, les bibliothécaires pourraient offrir aux enseignants des moyens d'intégrer les livres numériques à leur exploitation de réseaux littéraires.

\section{Conclusion}

Le soutien des bibliothécaires scolaires visant à favoriser l'intégration de la littérature jeunesse en classe est indispensable. La construction d'une vaste culture littéraire comme celle de Robert demande pour être initiée l'étayage de passionnés qui savent communiquer leur intérêt envers les œuvres. Malgré un attrait croissant pour les œuvres littéraires en classe, d'après nos observations lors de la formation initiale ou continue, la culture littéraire de la majorité des enseignants demeure limitée et leur usage des œuvres consiste encore souvent à des lectures à haute voix ponctuelles dont la visée concerne essentiellement l'accroissement de la motivation à lire.

La prise de conscience que la mise en réseau des œuvres apporte plus aux élèves que la lecture d'œuvres isolées est importante, de même que de savoir que ces mises en lien peuvent reposer sur des motifs variés. La capacité d'identifier à partir d'une ouvre donnée les activités susceptibles d'être réalisées avec les élèves afin de soutenir le développement de leurs compétences langagières et disciplinaires est influencée par le modelage opéré par la présentation de suggestions de réseaux.

Ainsi, donner accès et mettre en valeur dans les bibliothèques scolaires des réseaux variés destinés aux élèves de cycles différents, en s'inspirant par exemple de ceux présentés dans la revue Le pollen, est une action relativement simple qui est susceptible d'accroître l'intégration des œuvres littéraires en classe en réduisant plusieurs des obstacles que nous avons décrits. $\odot$

\section{Sources consultées}

Baillargeon, Claire, Marie-Hélène Charest, Martine Fortin \& Brigitte Moreau. 2014. La bibliothèque scolaire : les défis du XXI' ${ }^{\mathrm{e}}$ siècle. <apsds.org/wp-content/uploads/ APSDS_memoire_SRC_version_finale.pdf $>$ (consulté le 20 novembre 2014).

Boutevin, Christine \& Patricia Richard-Principalli. 2008. Dictionnaire de la littérature de jeunesse. Paris : Vuibert.

Chirouter, Edwige. 2007. Lire, réfléchir et débattre à l'école élémentaire. Paris: Hachette.

Devanne, Bernard. 2006a. Mettre en projet les apprentissages littéraires. Québec français (143) : 68-70.

Devanne, Bernard. 2006b. Lire, dire, écrire en réseaux : des conduites culturelles. Paris : Bordas.

Dupin de Saint-André, Marie \& Isabelle Montésinos-Gelet. 2009. Étude des pratiques d'une enseignante experte qui favorisent l'appropriation de l'écrit de ses élèves à la maternelle. Caractères (33) : 11-15.

Dupin de Saint-André, Marie \& Isabelle Montésinos-Gelet. 2012. Étude de cas des pratiques d'une enseignante experte et de leur influence sur le rapport à l'écrit d'élèves issus d'un milieu défavorisé. In Le rapport à l'écrit : habitus culturel et diversité, sous la direction de Carole Fleuret \& Isabelle MontésinosGelet. Québec : Presses de l'Université du Québec, 61-90.

Duprey, Sophie \& Gaëtan Duprey. 20o6. Parcours lectures de 4 à 7 ans : 14 parcours pour se construire une première culture littéraire et pour découvrir le monde. Schiltigheim : Accès Éditions.

Fédération des professionnelles et professionnels de l'éducation du Québec (FPPE-CSQ). 2013. Enquête sur la situation des bibliothèques scolaires. <www.fppe.qc.ca/index_doc/ ENQUETE BIBLIOTHEQUES_VF.pdf> (consulté le 20 novembre 2014).

Hall, Kathy. 2003. Effective literacy teaching in the early years of school: A review of evidence. In Handbook of Early Childhood Literacy, sous la direction de Joanne Larson \& Jackie Marsh. London : SAGE, 315-326. 
Ministère de l'Éducation, du Loisir et du Sport (MELS). 2001. Programme de formation de l'école québécoise. Québec: Gouvernement du Québec.

Ministère de l'Éducation, du Loisir et du Sport (MELS). 2007. Programme d'éthique et de culture religieuse. Québec: Gouvernement du Québec.

Ministère de l'Éducation, du Loisir et du Sport (MELS). 2009. Progression des apprentissages au primaire - Français langue d'enseignement. Québec: Gouvernement du Québec.

Montésinos-Gelet, Isabelle \& Marie-France Morin. 2007. Approcher l'écrit à pas de loup. La littérature de jeunesse pour apprendre à lire et à écrire au préscolaire et au primaire. Montréal : Chenelière Éducation.

Moreau, Brigitte. 2013. Profession : bibliothécaire scolaire. Formation et profession 21 (2) : 110-113.

Morin, Marie-France \& Lucie Roger. 2014. Les réseaux littéraires : clé du passeur culturel. Québec français (171) : 95-97.

Nadon, Yves. 2007. Écrire au primaire : réflexions et pratiques. Montréal : Chenelière.

Nadon, Yves. 2011. Apprendre à lire et à écrire en première année et pour le reste de sa vie ( $2^{\mathrm{e}}$ éd.). Montréal : Chenelière.

Olness, Rebecca. 2005. Using Literature to Enhance Writing Instruction: A Guide for $\mathrm{K}-5$ Teachers. Newark, DE : International Reading Association.

Schneider, Jean-Bernard. 2008. Projet lecteur : 11 parcours à travers la littérature de jeunesse -8 à 12 ans. Schiltigheim : Accès Éditions.

Tauveron, Catherine. 2002. Lire la littérature à l'école. Paris : Hatier.

Tauveron, Catherine. 2004. Fonctions et natures des lectures en réseaux. Éduscol. <eduscol.education.fr/cid46319/ fonctions-et-nature-des-lectures-en-reseaux.html > (consulté le 30 juin 2014). 